\title{
EL LABORATORIO DE INVESTIGACIÓN Y SU APORTE EN FAVOR DE LA BIODIVERSIDAD COSTARRICENSE
}

\section{(c) (1) $\Theta(9)$}

Dr.Misael Chinchilla Carmona P.H.D. ${ }^{1}$

Dra. Idalia Valerio Campos M.Q.C. ${ }^{2}$

1. Encargado de Investigación UCIMED

2. Especialista en Parasitología / Investigación UCIMED.

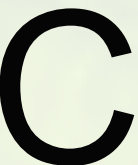

osta Rica es un país privilegiado en cuanto a su abundante flora y fauna la cual alberga en sus ricos nichos ecológicos una exuberante naturaleza de la que nos preciamos a nivel internacional. A pesar de ser un país pequeño en donde realmente en pocas horas, podemos ir de costa a costa y de frontera a frontera, la cantidad de especies de plantas y de animales que poseemos constituyen un verdadero ejemplo de naturaleza viviente. De esto podemos dar fe quienes por razón de nuestros estudios, que luego resumiremos, hemos tenido el privilegio de observar.

El Laboratorio de Investigación de la UCIMED ha querido contribuir, aunque sea en muy pequeña escala, con la preservación de este tesoro natural, realizando estudios de la endofauna de varios grupos de animales. Con este objetivo en mente, nos hemos dado a la tarea de identificar los géneros y especies de parásitos presentes en los animales estudiados, a fin de que basados en esos conocimientos, veterinarios y demás servidores en el campo, apliquen los tratamientos correspondientes tanto en centros de rescate como en zoológicos $u$ otros centros similares. Colateralmente, estos análisis nos han permitido demostrar la presencia de parásitos que eventualmente pueden también infectar al ser humano, lo que obviamente es muy importante desde un punto de vista médico.

A continuación, algunos de los estudios realizados que se citan secuencialmente de acuerdo con los temas abordados.

\section{Estudios en monos (primates).}

Estos fueron los primeros estudios con que se inició la investigación en esta universidad y en conjunto con investigadores de la Universidad de Costa Rica Así, estudiamos los parásitos intestinales de las cuatro especies de este grupo que existen en nuestro país. Los animales estudiados fueron el mono aullador (Alouatta palliata) (Chinchilla et al. 2005), el carablanca (Cebus capucinus) estudio general (Chinchilla et al.
Recibido: 2019-04-01

Publicado: 2019-04-29
2007) y específicamente en una área protegida Limón (Chinchilla et al. 2010), el ardilla o tití (Saimiri oerstedii) (Chinchilla et al. 2010) y el araña (Ateles geoffroyi) (Chinchilla et al. 2010). En todos ellos se encontraron varias especies de organismos, algunos, como el caso de los anquilostomidios que pueden causar una importante patología en el animal. En los monos Congo o aulladores también se encontraron en la sangre tres parásitos interesantes cuyo reporte previo no se conocía: el Trypanosoma minasense (Chinchilla et al. 2005), Plasmodium brasilianum un organismo también reportado en humanos y cuya morfología es idéntica a $P$. malariae, Trypanosoma rangeli un parásito que aunque no es patógeno, también se ha encontrado en el ser humano (Valerio et al. 2012)

Dentro del estudio de estos primates se hizo un intenso análisis estadístico de la morfometría (medidas de todas las partes de la estructura del animal) de las cuatro especies, análisis en que el trabajo del estadístico Juan Carlos Vanegas fue intenso y clave para completar estos estudios (Sanchez et al. 2014, 2018, 2018a).

\section{Estudios en cánidos}

Como parte de una tesis de maestría de la estudiante Carmen Niehaus se tuvo oportunidad de analizar muestras de heces de coyotes (Canis latrans) de la región cercana al Volcán Irazú, encontrándose varios parásitos intestinales, algunos de los cuales pueden, eventualmente infectar al ser humano, al menos como un parasitismo extraviado (Caso de Toxocara sp.) (Niehaus et al. 2012). También se encontraron en estos animales protozoarios de los géneros Cryptosporidium y Cyclospora asociados en los humanos con problemas de inmunosupresión (Niehaus et al.211). Este mismo tipo de organismos fue diagnosticado en este laboratorio en perros del área Metropolitana de Costa Rica (Valerio et al.2010). 


\section{Parasitismo en felinos}

En este caso, ya se publicó un estudio de los parásitos intestinales de varios felinos (Leopardus wiedii, $L$. pardalis, Panthera onca, P. leo, Puma concolor y Puma yagouaroundi) en cautiverio en el Centro de Rescate para la Vida Silvestre La Marina-Costa Rica (Chinchilla et al. 2009) Dentro de los organismos encontrados resalta la presencia de huevecillos de Paragonimus, sp. y ooquistes de Toxoplasma gondii, ambos parásitos importantes del ser humano. También Valerio et al. (2005) han reportado la presencia de Spirometra mansoni en un gato casero. Más recientemente hemos estudiado alrededor de 900 muestras de heces de felinos silvestres trabajo que será publicado prontamente (Chinchilla et al. 2019) (en preparación). En este caso, también se han encontrado algunos parásitos importantes para el ser humano, tales como los antes indicados y algunos otros, incluso aquellos que se relacionan con procesos de inmunosupresión. Un análisis estadístico completo fue realizado también por el estadístico Juan Carlos Vanegas

\section{Investigaciones en roedores}

En el estudio de las heces de ciertos roedores (Melanomys caliginosus, Heteromys desmarestianus, Peromyscus mexicanus, Tylomys watsoni y de un marsupial Philander opposum) se encontraron varios parásitos, algunos de los cuales podrían causar importantes lesiones en el animal, además de organismos presentes en la sangre que también son importantes ( Chinchilla et al. 2013) Dentro de los intestinales, se reportaron dos especies del género Vexillata, uno ya conocido y otro que se reporta por primera vez en nuestro país (Chinchilla et al. 2014) En el caso del análisis sanguíneo, se reportó la presencia de Babesia sp y Bartonella sp organismos importantes para la salud de estos roedores (Chinchilla et al. 2016).Finalmente en el roedor Melanomys caliginosus hemos reportado tres especies nuevas a nivel mundial de protozoarios del genero Eimeria (E.melanomytis, E. caliginosa y E. rebambensis) (Chinchilla et al.2013, 2014). Se han realizado, además, los ciclos evolutivos de los dos primeros, así como de E. marmosopos, un parásito de zorro pelón antes descrito (Chinchilla et al. 2015, 2016, 2018)

Como siempre lo he dicho, todo el trabajo realizado en nuestro laboratorio no hubiera sido posible si no contáramos con un personal calificado pero sobre todo impregnado de una mística, espíritu de trabajo y valores humanos que estimula a los suscritos a continuar luchando por ideales, a veces difíciles de cumplir, pero siempre presentes como parte de nuestra meta para el mejor futuro de la institución a quien nos debemos.
BIBLIOGRAFÍA

I.Valerio l., B. Radríguez y M. Chinchilla. 2005. Primer hallazgo de Spirametra mansani en Felis domesticus de Costa Rica. Parasitol. Latinaam. 59: I62-166.

2.Chinchilla, M., A. Troya, Q.M. Guerrero, G. Gutiérrez y R. Sánchez. 2005. Presencia de Trypanasoma minasense (Kinetoplastida: Trypanasamatidae) en Alouatta palliata (Primates: Cebidae) de Costa Rica. Parasitol. Latinoam. 60:90-92.

3.Chinchilla, M., Q. M. Guerrera, G. A. Gutiérrez-Espeleta, R. Sánchez \& B. Radríguez. 2005. Parásitas intestinales en monos congo Alauatta palliata (Primates: Cebidae) de Casta Rica. Rev. Biol. Trap. 53: 437-445.

4.Chinchilla, M., Q.M. Guerrero, G. Gutiérrez, R. Sánchez. 2006. Presencia de Plasmodium brasilianum (Apicamplexa, Plasmodidae) en el mona congo (Alouatta palliata, Primates: Cebidae) de Costa Rica. Importancia epidemológica en relación con el ser humano. Parasitol. Latinoam bi: 192-196.

5.Chinchilla, M., Guerrero, Q., Gutiérrez-Espeleta, G. \& Valerio, I. 2007. Parásitas intestinales en monos carablanca Cebus capucinus (Primates: Cebidae) de Costa Rica. Parasital Latinoam. 62: 170-175.

G.Valerio, I, Ulate, R.., Sota, M. y Chinchilla, M. 20ID. Hallazgo de parásitas oportunistas en perros (Canis familiaris) del área metropolitana de Casta Rica. Rev. Ibero-Latinam. Parasital. 69 (I):52 59.

7.Chinchilla, M. Valerio, I. Guerrero. DM.. Gutiérrez, G. y Sánchez, R. 201D a. Infección po parásitos intestinales de monos araña Ateles geaffrayi (Primates: Cebidae) en cautiverio de Costa Rica. Rev. Ibero-Latinam. Parasital. 69 (I):IDI-I05.

8.Chinchilla, M.., Valerio, I., Guerrero, OM., Gutiérrez, G. y Sánchez, R. 20ID. Parasitismo intestina en manas tití a ardilla Saimiri aerstedii (Primates: Cebidae) de Costa Rica. Rev. lbera-Latinam. Parasitol. 69 (I):IQ6-III.

9.Chinchilla, M., Urbani, B., Valerio, I. y Vanegas, JC. 20I0. Parasitasis intestinal en monos capuchinos cariblancos Cebus capucinus (Primates: Cebidae) de un área protegida en la provincia de Limón, noreste de Casta Rica. Rev. Biol. Trap. Vol. 58 (4): 1335-1346. I0.Niehaus, L., Valerio, I., Blanco, K. \& Chinchilla, M. 20II. Presencia de protozoarios y microorganismos relacionados con procesas de inmunosupresión humana en coyotes (Lanis latrans: Canidae) del Parque Nacional Volcán Irazú y campo agrícola limítrafe en Costa Rica. Rev. Ibero-Latinaam. Parasital. 70(2): 197-205.

II.Niehaus, C., Valerio, I., Blanca, K. \& Chinchilla, M. 2012. Infecciones parasitarias del cayote, Canis latrans (Carnivora: Canidae) en un Parque Nacional y una zona agrí́cola en Costa Rica. Rev. Bial. Trop. 60 (2): 799-808.

12.Valerio, I., Chinchilla, M., Sánchez, R. \& Gutiérrez-Espeleta, G. 2012. El mano aulladar

Alouatta palliata (Primates:Lebidae) nuevo reservario de Trypanosoma rangeli

(Trypanosomatida:Trypanasomatidae) en Costa Rica. Brenesia. 78:96-99.

13.Chinchilla-Carmona, M.., Valerio-Campos, I., Sánchez-Porras, R., González-Paniagua, A.

Martínez-Esquivel, L. \& Dusynszki, D. 2013. Two new Eimeria spp. (Apicamplexa: Eimeriidae) from the dusky rice rat, Melanomys caliginosus, Tome 1860, in Costa Rica. The Journal of Parasitology 99(I): $82-84$.

14Chinchilla-Carmana, M., Valerio-Campos, I., Sánchez-Porras, R., Martínez-Esquivel, L., GonzálezPaniagua, A., Valerio-Campos, L., Bolaños-Jiménez, J. \& León-Ganzález, L. Parásitos intestinales y sanguíneos de 4 especies de raedares y 5 ejemplares de Philander apossum (Didelphimarphia: Didelphidae) capturados en la Reserva Biológica Alberto Manuel Brenes (REBAMB) de Costa Rica. Rev. Ibero-Latinoam. Parasital. (2013); 72 (2): $176-184$

15.Chinchilla-Carmona, M., Valerio-Campos, I., Sánchez-Porras, R., González-Paniagua, A. \& Dusynszki, D. 2014. A new species of Eimeria Schneider, 1875 (Apicamplexa: Eimeriidae) fram the dusky rice rat Melanomys caliginosus (Tomes) (Mammalia: Radentia) in Costa Rica. Systematic Parasitology 88(2): 181-184.

I6.Chinchilla-Carmona, M., Valerio-Campos, I. \& Sánchez-Porras. 2014. Presencia de dos especies de Vexillata (Nematada: Ornithostrangylidae) en Heteromys desmarestianus (Rodentia: Heteromyidae) de la Reserva Biológica Alberto Manuel Brenes, San Ramńn, Costa Rica. Brenesia 81-82: 130-133.

17.Chinchilla, M., Valerio, I., Duszynski, D.W. 20I5. Endogenous life cycle of Eimeria marmosaps (Apicomplexa: Eimeriidae) from the opossum Didelphis marsupialis (Didelphimarphia: Didelphidae). Journal of Parasitology IDI: 436-444.

18.Chinchilla, M.. Valerio, \& Duszynski, D.W 2016. Endagenous life cycle of Eimeria melanomytis (Aplicomplexa: Eimeriidae) from the dusty rice rat, Melanomytis caliginosus (Rodentia: Cricetidae: Sigmodontinae) in Costa Rica. J. Parasital IDZ(I)

19.15.Chinchilla-Carmana, M., Valerio-Campos, I., Sánchez-Porras, R, Valerio L \& J Bolaños. (20I6) Presencia de Babesia sp. (Piroplasmida: Babesidae) in Melanomys caliginasus (Rodentia: Critidae) de una reserva biológica de Costa Rica. Brenesia 85-86: 82-85, 2016

20.Sánchez-Porras, R., Chinchilla-Carmona, M., Valerio-Campos, I. \& Vanegas-Pissa, JL. 2018.

Aspectos morfamétricos del mono carablanca Cebus imitadar y del mano ardilla Saimiri aerstedi (Primates: Cebidae) de Costa Rica. La primatología en América Latina. Ediciones IVIC. Instituto de Investigaciones Cientificas (IVIC)

21.Chinchilla, M.., Valerio, \& Duszynski, D.W. 2018. Intestinal Cycle of Eimeria Caliginosa (Apicamplexa: Eimeridae) from the dusky rice rat, Melanomys Caliganosus (Rodentia: Cricetidae: Sigmadontinae), in Costa Rica. J. Parasital., I04 (4), pp: 347-352. 